International Journal of Pharmaceutics \& Pharmacology

EDW SER

Available Online: http://ijpp.edwiserinternational.com

\title{
Liposomal Nanoformulation of Piperlongumine for Improved Aqueous Solubility and Enhanced Anti-tumour Activity In-vitro
}

\author{
Ali Alamri ${ }^{1}$, Yanlan Huang ${ }^{l}$ and Hongwei Zhang ${ }^{1 *}$ \\ ${ }^{1}$ Department of Pharmaceutical Sciences, School of Pharmacy, MCPHS University, Boston, MA 02115, USA
}

Article info

Received 13 December 2018

Revised 26 December 2018

Published 31 December 2018

*Corresponding author: Hongwei

Zhang, Department of Pharmaceutical Sciences, School of Pharmacy, MCPHS University, Boston, MA 02115, USA; E-mail: Hongwei.Zhang@mcphs.edu

\begin{abstract}
Piperlongumine (PL) is an alkaloid isolated from the Piper species, which can induce apoptosis in cancer cells but has little inhibitory effect on the growth of normal cells. However, its poor aqueous solubility has largely limited its therapeutic applications. To address this issue, we developed liposomal nanoformulations for PL (nanoPLs) in this study. The optimized nanoPLs exhibited a small average diameter in the range of 100-160 nm and narrow size distribution as well as high encapsulation efficiency. NanoPLs also demonstrated excellent storage stability at $4{ }^{\circ} \mathrm{C}$ for 12 weeks. The apparent solubility of PL in NanoPLs was improved up to 312 folds than in PBS. NanoPLs also markedly increased the cytotoxicity of PL to human prostate cancer PC-3 cells when compared to free PL. NanoPLs represent promising and convenient injectable formulations of $P L$ for further preclinical and clinical investigation.
\end{abstract}

Keywords: Piperlongumine; Nanoparticles; Liposomes; Cancer; Solubility; Cytotoxicity

\section{Introduction}

Piperlongumine (PL, also known as piplartine, CAS\#: 20069-09-4, 5,6-dihydro-1-[(2E)-1-oxo-3-(3,4,5trimethoxyphenyl)-2-propenyl]-2(1H)-pyridinone,

Figure 1) is a bioactive alkaloid isolated from black pepper (Piper nigrum) and long pepper (P. longum) [1]. Previous studies have revealed that PL has different pharmacological effects, including anti-diabetic, antifungal, anti-leishmania, anti-inflammatory, anti-platelet aggregation, and anti-cancer activities [2]. Recently, PL has attracted special attention as a potential antineoplastic agent because it showed selective cytotoxicity to a plethora of human cancer cells, including pancreatic, breast, lung, colon and bladder cancer, but not to normal cells [3]. Piperlongumine increases the level of reactive oxygen species (ROS) and promotes apoptosis in cancer cells [4]. The selectivity of PL for cancer cells over normal cells is related to the over-expression of nuclear factor erythroid 2-related factor 2 (Nrf2) in cancer cells [5].
Piperlongumine binds to and inhibits the activity of Nrf2, which is an essential component of the cell to regulate the antioxidants activity, resulting in the reduction of intracellular antioxidants. It was also shown that there are alternative mechanisms of action, including the induction of autophagy in cancer cells by targeting p38 signaling [6] and inhibitory effects on STAT3, which is independent of the ROS mechanism [7].

Despite such appealing potential in cancer treatment, the therapeutic application of PL has yet been embraced mainly due to its strong hydrophobicity and extremely poor aqueous solubility, which has been determined separately by Aodah et al. $(0.026 \mathrm{mg} / \mathrm{mL})$ [8] and Fofaria et al. $(0.32 \mathrm{mg} / \mathrm{mL})$ [9]. When dispersed in a physiological solution, PL easily forms aggregates, resulting in the formation of a turbid suspension, but not a solution. Intravenous administration of PL suspension 
Citation: Alamri A, Huang Y, Zhang H. Liposomal Nanoformulation of Piperlongumine for Improved Aqueous Solubility and Enhanced Anti-tumour Activity In-vitro. Int J Pharm Pharmacol 2018; 2: 133. doi: $\underline{10.31531 / 2581-3080.1000133}$

to tumour-bearing animals or to cancer patients will inevitably result in embolism which may be fatal [10, $11]$.

In addition, the poor aqueous solubility made it impossible for dose escalation in disease treatment. Although Cremophor EL and other vehicles are used in clinic to address the solubility issue of certain therapeutic agents, such as paclitaxel, many problems have been identified with the use of the vehicles, such as peripheral neuropathy, aggregation of erythrocytes, hyperlipidaemia and severe anaphylactoid hypersensitivity reactions [12-16]. Therefore, it is of great importance to develop a delivery system that can significantly increase the solubility of PL to enable parenteral administration in a physiological solution.

Previously, PL has been formulated into chitosan nanoparticles [17], polymeric micelles [5,18], PLGA nanoparticles [19] and nanoemulsions [9] to solve the solubility issue. However, the apparent solubility of PL in these formulations was not reported in any of these nanoformulation. Thus, it is unclear whether these formulations are suitable for intravenous administration. Injectable liposomal nanoformulations for PL delivery have yet been reported. Liposomes are small artificial lipid vesicles consisting of hydrophilic and hydrophobic regions. The aqueous pool of liposomes is surrounded by bilayers of lipids which are composed of natural or synthetic phospholipids. Liposomes have significant advantages in drug delivery to improve the aqueous solubility and stability of therapeutic agents [20-24].

Through the hydrophobic interaction, hydrophobic compounds can be incorporated into the non-polar lipid bilayers of liposomes. Thus, the aqueous solubility of the poorly water-soluble compound can be dramatically improved. In the past several decades, liposomal nanotechnology has helped a number of poorly soluble hydrophobic drugs reach market, such as paclitaxel [25] and irinotecan [26,27]. It also helped numerous promising therapeutic molecules advance to clinical/preclinical investigation, such as ibuprofen [22] and indomethacin [28]. From clinical oncology point of view, by limiting the particle size to approximate 100$200 \mathrm{~nm}$, liposomes can preferentially accumulate in solid tumour tissues after intravenous administration by means of the "enhanced permeation and retention" (EPR) effect [29-31], and decrease their distribution to normal tissues [32]. In fact, a number of reports have demonstrated enhanced drug concentration in the solid tumour tissue delivered by nanoliposomes than by drug solution $[33,34]$.

Thus, nanoliposomes can further enhance the therapeutic index as well as the antitumor specificity of PL. In this work, we report the preparation, physicochemical characterization, storage stability and cytotoxicity of liposomal nanoformulations of PL (nanoPLs). For the first time, PL was successfully formulated into stable liposomal nanoparticles which markedly improved its aqueous solubility. In addition, nanoPLs exhibited stronger ability to induce cancer cell death compared to free PL.

\section{Materials and Methods}

\section{Materials}

Phosphatidylcholine (PC), N-[1-(2, 3-Dioleoyloxy) propyl]-N, N, N-trimethylammonium methyl-sulphate (DOTAP) and cholesterol (Chol) were purchased from Avanti Polar Lipids (Alabaster, AL). Piperlongumine (PL) was purchased from Cayman Chemical Co. (Ann Arbor, MI). Phosphate buffer solution (PBS, pH 7.4) was purchased from VWR (Radnor, PA).

Cell culture media (Kaighn's Modification of Ham's F12k Medium), Trypsin-EDTA Solution, and PenicillinStreptomycin Solution were purchased from American Type Culture Collection (ATCC) (Manassas, VA). CellTiter $96^{\circledR}$ AQueous One Solution Cell Proliferation Assay kit was purchased from Promega (Madison, WI).

\section{Cell Culture}

Human prostate cancer PC-3 cells were purchased from ATCC (Manassas, VA). The cells were maintained in Kaighn's Modification of Ham's F-12k Medium supplemented with $10 \%$ FBS in a humidified $\mathrm{CO} 2$ incubator at $37^{\circ} \mathrm{C}$.

\section{Preparation of PL-loaded Liposomal Nanoformulations (NanoPLs)}

NanoPLs were prepared using the thin film rehydration method followed by sonication [35]. Briefly, varied amount of PL and lipid materials containing PC, DOTAP and cholesterol at a molar ratio of 60/30/10 were dissolved in $4 \mathrm{~mL}$ of chloroform in a round bottom flask. The organic solvent was completely removed on a rotary evaporator to obtain a thin lipid film in the flask. The dry lipid film was hydrated with PBS to form a crude liposome suspension, and then the suspension was 
Citation: Alamri A, Huang Y, Zhang H. Liposomal Nanoformulation of Piperlongumine for Improved Aqueous Solubility and Enhanced Anti-tumour Activity In-vitro. Int J Pharm Pharmacol 2018; 2: 133. doi: $\underline{10.31531 / 2581-3080.1000133}$

transferred to a glass tube and kept in a refrigerator at $4^{\circ} \mathrm{C}$ overnight.

The crude liposome suspension was then sonicated at $55^{\circ} \mathrm{C}$. The cholesterol percentage remained at $10 \%$ (molar \%) while the percentage of DOTAP varied at $30 \%, 40 \%$ and $50 \%$ of the total lipid materials. The ratio of PL to total lipid materials was optimized at 1/10, $1.5 / 10$ and 2/10 (molar ratio). For formulations containing $40 \%$ and $50 \%$ DOTAP, the percentage of PC was lowered to $50 \%$ and $40 \%$, respectively. In total, nine different nanoPLs were prepared. Three blank liposomal vehicles which do not contain PL were prepared in the same manner.

\section{Physicochemical Characterization of NanoPLs}

The average particle size and size distribution were measured by dynamic light scattering. A Brookhaven NanoBrook 90PLUS particle sizer and zeta potential analyser was used to determine the average size, zeta potential, and polydispersity index (PDI). The samples were diluted in PBS ( $\mathrm{pH} 7.4$ ) by 5 folds prior to the measurement. The intensity of scattered light was detected at $90^{\circ}$ angle to an incident beam. All the data analysis was performed in automatic mode.

\section{HPLC Analysis}

PL was analysed using high performance liquid chromatography (HPLC). The HPLC system (Agilent Technologies, Santa Clara, CA) consisted of a G4212B diode Array detector, a G1311B/C quaternary pump, tow plunger chambers and an Agilent Lab Advisor software. The analysis was performed with a ZOPRAX Extended C-18 (150 mm, 5 microns) column which was maintained at room temperature.

The mobile phase consisted of $70 \%$ (v/v) methanol and $30 \%(\mathrm{v} / \mathrm{v})$ distilled water with the $\mathrm{pH}$ being adjusted to 8.1. The flow rate was $1 \mathrm{~mL} / \mathrm{min}$ and the injection volume was $20 \mu \mathrm{L}$. The UV absorbance from PL was measured at $253 \mathrm{~nm}$ wavelength [36].

\section{Solubility Study}

To determine the solubility of PL in PBS ( $\mathrm{pH} 7.4$ ), excessive amount of PL was added to $1 \mathrm{~mL}$ of PBS followed by constant shaking for $24 \mathrm{~h}$ at room temperature. Then the PL suspension was visually checked, and the presence of undissolved PL aggregates was confirmed. PL suspension was centrifuged at 11400 $\mathrm{g}$ for $5 \mathrm{~min}$ where the undissolved and aggregated PL settled down. The supernatant was diluted in methanol and filtered by syringe filters with the pore size of 0.22 $\mu \mathrm{m}$ prior to analysis by HPLC. For nanoPLs, unencapsulated PL aggregates were removed using the same centrifugation condition. The concentration of PL in the supernatant was determined by HPLC as described above.

\section{Encapsulation Efficiency and Drug Loading Efficiency Determination}

NanoPLs were centrifuged at $11400 \mathrm{~g}$ for $5 \mathrm{~min}$ at $4^{\circ} \mathrm{C}$ to separate free PL from nanoPLs. The supernatant was carefully taken out from the Eppendorf tube and diluted by methanol, and the concentration of PL in the supernatant was determined by HPLC. The encapsulation efficiency (EE\%) was calculated by the following equation:

$\mathrm{EE} \%=($ The amount of PL in nanoPLs $) /($ Total amount of PL added to prepare nanoPLs) $\times 100$

The drug loading efficiency (DL\%) of nanoPLs was analysed after removing water from the nanoPLs by freeze-drying. The unencapsulated PL aggregates was separated by centrifugation, then nanoPLs supernatant was transferred to a weighted empty glass vial followed by lyophilization. The exact weight of nanoPLs was calculated by deducting the weight of empty glass vial from the weight of the glass vial containing lyophilized nanoPLs. Subsequently, the nanoPLs were dissolved in methanol and the concentration of PL was determined using HPLC. The following equation was used to calculate DL\%:

DL\% $=($ The weight of PL in nanoPLs $) /($ The weight of nanoPLs) $\times 100$

\section{Physical Stability Study}

NanoPLs were sealed in glass vials and stored at $4^{\circ} \mathrm{C}$. The particle size and PDI were analysed every 4 weeks for a period of 12 weeks. EE \% and DL \% were measured immediately after preparation (week 0 ) and after 12 weeks (week 12).

\section{In vitro Antitumor Activity}

Cytotoxicity analysis was performed using a CellTiter 96 ${ }^{\circledR}$ AQueous Cell Proliferation Assay kit. Briefly, human prostate cancer PC-3 cells were seeded in a 96well plate at a density of $1 \times 10^{4}$ cells per well 24 hours prior to the treatment. Then the cells were treated with 
Citation: Alamri A, Huang Y, Zhang H. Liposomal Nanoformulation of Piperlongumine for Improved Aqueous Solubility and Enhanced Anti-tumour Activity In-vitro. Int J Pharm Pharmacol 2018; 2: 133. doi: $\underline{10.31531 / 2581-3080.1000133}$

free PL (in DMSO) or nanoPLs with the final PL concentrations at $3,6,12,18,24$, and $30 \mu \mathrm{M}$ in each well for 24 hours.

When the treatment was finished, the relative cell viability compared to non-treated cells was measured using the kit according to the manufacturer's protocol. The absorbance was taken at $490 \mathrm{~nm}$ using a microplate reader. All experiments were set up in triplicates to determine means and standard deviations. The percentage of viable cells was calculated as follows:

Cell viability $(\%)=($ Absorbance intensity of treated cells $) /($ Absorbance intensity of non-treated cells $) \times 100$ (3)

To determine the half maximal inhibitory concentration (IC50) values of PL and nanoPLs to PC-3 cells, the cells were seeded at $1 \times 10^{4}$ cells per well in a 96 well plate 24 hours prior to the treatment. The cells were exposed to free PL and nanoPLs at different concentrations $(0.01,0.1,1,10$ and $100 \mu \mathrm{M})$ for 24 hours, then the cell viability was determined using the cell proliferation assay kit. The IC50 values of free PL and nanoPLs against PC-3 cells were calculated using GraphPad Prism [37].

\section{Statistical Analysis}

Data were presented as mean \pm standard deviation. GraphPad Prism software was used for statistical analysis. For comparison between two groups, linear regression with $95 \%$ confidence interval and unpaired two-tailed Students' $t$ test was performed. For comparison of paired data among three or more groups, one-way analysis of variance (ANOVA) followed by a Tukey's post hoc analysis was used. Difference was considered statistically significant when a value of $\mathrm{p}<0.05$.

\section{Results}

\section{Characterization of PL-loaded liposomal nanoformulations (nanoPLs)}

To prepare nanoPLs, the simple yet classic thin film rehydration followed by sonication method [35] was used. According to this method, PL was first mixed with lipid materials to form a thin and dry film, and then the film was re-dispersed in PBS. Nine different liposomal nanoformulations containing PL were prepared to investigate the influence of the DOTAP percentage in the lipid materials and the ratio of PL to total lipid materials on the characterization of nanoPLs. The particle size, size distribution and surface charge of nanoPLs are summarized in Table 1 . To evaluate the effect of DOTAP\% on the physicochemical characteristics of the nanoPLs, the cholesterol \% remained unchanged at $10 \%$ by molar. Dynamic light scattering data showed that when the ratio of PL to total lipid materials was fixed at $1 / 10$ (molar ratio), increasing the DOTAP percentage resulted in the decrease of average particle size from $160.4 \pm 7.7 \mathrm{~nm}$ (30\% DOTAP, formulation 1C) to $123.1 \pm 7.6 \mathrm{~nm} \mathrm{(40 \%}$ DOTAP, formulation 2C), and further decrease to 102.9 $\pm 2.4 \mathrm{~nm}$ (50\% DOTAP, formulation 3C). The similar decrease was observed when the ratio of PL to total lipid materials was 1.5/10. However, when the ratio of PL to total lipid materials was $2 / 10$, altering DOTAP\% did not significantly reduce the particle size of nanoPLs. The surface charge of nanoPLs was also increased from 18.9 $\pm 9.01 \mathrm{mV}$ (formulation 1C) to $26.9 \pm 8.20 \mathrm{mV}$ (formulation 2C), then further to $49.8 \pm 15.40 \mathrm{mV}$ (formulation 3C) when the percentage of DOTAP was increased from $30 \%$ to $40 \%$ and $50 \%$.

When the PC/DOTAP/Chol is fixed at 40/50/10 (molar ratio), lowering the ratio of PL to total lipid materials significantly decreased the average particle size from $263.4 \pm 30.2 \mathrm{~nm}$ (formulation $3 \mathrm{~A}$ ) to $217.0 \pm 16.5 \mathrm{~nm}$ (formulation 3B) then to $102.9 \pm 2.4 \mathrm{~nm}$ (formulation $3 \mathrm{C})$. Formulations $1 \mathrm{C}, 2 \mathrm{C}$, and $3 \mathrm{C}$ were chosen for further characterization as they represented smaller particle size as well as narrower size distribution (Table 1). Using HPLC, the encapsulation efficiency (EE\%) of formulation $1 \mathrm{C}, 2 \mathrm{C}$ and $3 \mathrm{C}$ was determined to be 93.8 $\pm 0.04,95.2 \pm 0.01$, and $97.5 \pm 0.03 \%$, respectively, and drug loading efficiency (DL\%) of the three formulations to be $2.3 \pm 0.03,2.6 \pm 0.18$, and $1.2 \pm 0.02 \%$, respectively (Table 2 ).

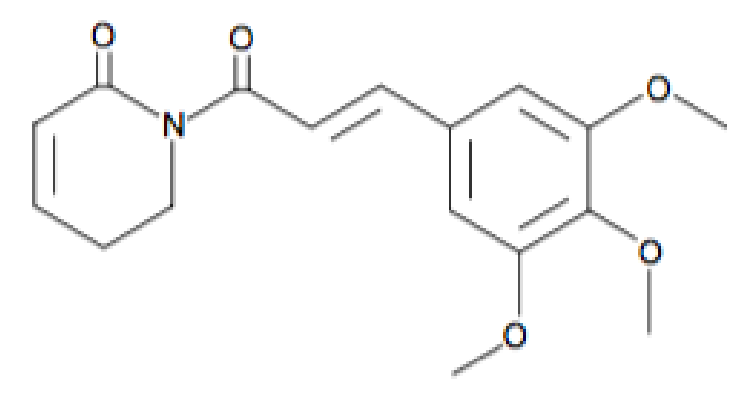

Figure 1: The chemical structure of piperlongumine. 
Citation: Alamri A, Huang Y, Zhang H. Liposomal Nanoformulation of Piperlongumine for Improved Aqueous Solubility and Enhanced Anti-tumour Activity In-vitro. Int J Pharm Pharmacol 2018; 2: 133. doi: $\underline{10.31531 / 2581-3080.1000133}$

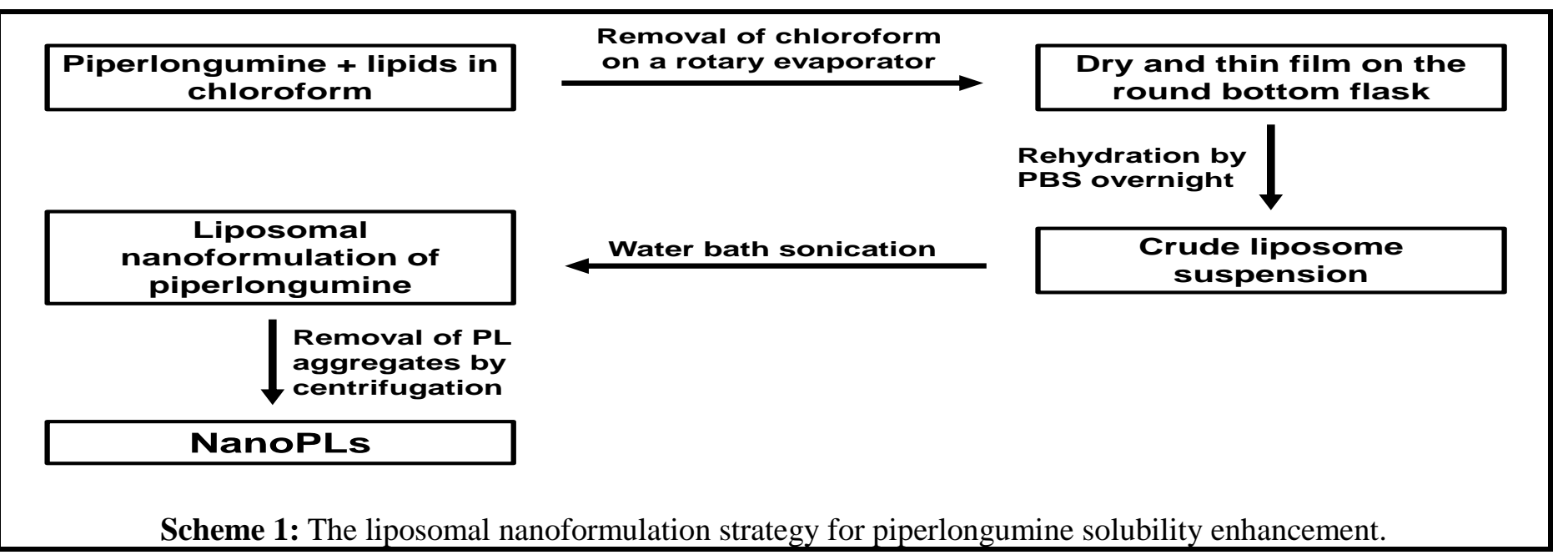

Table 1: NanoPLs: formulation composition, particle size and surface charge.

\begin{tabular}{|c|c|c|c|c|c|}
\hline $\begin{array}{c}\text { Formulati } \\
\text { on ID }\end{array}$ & $\begin{array}{c}\text { PC/DOTAP/Chol } \\
\text { (Molar ratio) }\end{array}$ & $\begin{array}{c}\text { PL to total lipid ratio } \\
\text { (Molar ratio) }\end{array}$ & Size (d. nm) & $\begin{array}{c}\text { Zeta } \\
\text { Polydispersity } \\
\text { index (PDI) }\end{array}$ & $\begin{array}{c}\text { potential } \\
\text { (mV) }\end{array}$ \\
\hline 1A & $60 / 30 / 10$ & $02-10$ & $268.1 \pm 52.8$ & $0.34 \pm 0.03$ & $9.3 \pm 3.30$ \\
\hline 1B & $60 / 30 / 10$ & $1.5 / 10$ & $280.8 \pm 7.6$ & $0.36 \pm 0.01$ & $27.7 \pm 1.40$ \\
\hline 1C & $60 / 30 / 10$ & $01-10$ & $160.4 \pm 7.7$ & $0.21 \pm 0.01$ & $18.91 \pm 9.01$ \\
\hline 2A & $50 / 40 / 10$ & $02-10$ & $277.9 \pm 32.7$ & $0.36 \pm 0.02$ & $24 \pm 0.60$ \\
\hline 2B & $50 / 40 / 10$ & $1.5 / 10$ & $247.6 \pm 20.9$ & $0.33 \pm 0.20$ & $32.3 \pm 4.40$ \\
\hline 2C & $50 / 40 / 10$ & $01-10$ & $123.1 \pm 7.6$ & $0.24 \pm 0.02$ & $26.9 \pm 8.20$ \\
\hline 3A & $40 / 50 / 10$ & $02-10$ & $263.4 \pm 30.2$ & $0.32 \pm 0.01$ & $37.8 \pm 4.90$ \\
\hline 3B & $40 / 50 / 10$ & $1.5 / 10$ & $217.0 \pm 16.5$ & $0.34 \pm 0.01$ & $29.6 \pm 16.40$ \\
\hline 3C & $40 / 50 / 10$ & $01-10$ & $102.9 \pm 2.4$ & $0.29 \pm 0.02$ & $49.8 \pm 15.40$ \\
\hline
\end{tabular}

Table 2: The encapsulation efficiency (EE\%) and drug loading efficiency (DL\%) of NanoPLs.

\begin{tabular}{|l|l|l|l|l|}
\hline \multirow{2}{*}{$\begin{array}{c}\text { Formulati } \\
\text { on ID }\end{array}$} & \multicolumn{2}{|c|}{ Time 0 } & \multicolumn{2}{c|}{ 12 weeks } \\
\cline { 2 - 5 } & EE\% & DL\% & EE\% & DL\% \\
\hline \multirow{2}{*}{$1 C$} & $93.83 \pm$ & $2.30 \pm$ & $94.09 \pm$ & $2.32 \pm$ \\
\hline & 0.04 & 0.03 & 0.02 & 0.05 \\
\hline $\mathrm{C}$ & $95.23 \pm$ & $2.38 \pm$ & $93.61 \pm$ & $2.33 \pm$ \\
\hline \multirow{3}{*}{$3 \mathrm{C}$} & 0.01 & 0.18 & 0.01 & 0.02 \\
\hline
\end{tabular}

Solubility of PL in liposomal nanoformulations

The apparent solubility of PL in PBS and in NanoPL 1C, 2C, 3C was determined after separating $\mathrm{PL}$ aggregates from nanoPLs by centrifugation. There were many undissolved flakes when the excessive amount of PL was dispersed in PBS. After centrifugation, a white pellet at the bottom of the Eppendorf tube was easily seen (Figure 2A, left). HPLC analysis data showed that the solubility of PL in PBS at pH 7.4 is only $0.02 \pm 0.01$ $\mathrm{mg} / \mathrm{mL}$. On the contrary, nanoPLs were almost clear and well-dispersed formulations. No visible pellets were observed after centrifugation (Figure 2A, right). The solubility of PL in 1C, 2C, and 3C formulations was $5.14 \pm 0.38 \mathrm{mg} / \mathrm{mL}, 6.25 \pm 0.44 \mathrm{mg} / \mathrm{mL}$ and $4.64 \pm 0.25$ $\mathrm{mg} / \mathrm{mL}$, respectively $(\mathrm{p}<0.05$, Figure $2 \mathrm{~B})$. The apparent solubility of PL in NanoPL 1C, 2C and 3C exceeded the intrinsic solubility of free PL in PBS by 257, 312 and 232 times, respectively. 
Citation: Alamri A, Huang Y, Zhang H. Liposomal Nanoformulation of Piperlongumine for Improved Aqueous Solubility and Enhanced Anti-tumour Activity In-vitro. Int J Pharm Pharmacol 2018; 2: 133. doi: $\underline{10.31531 / 2581-3080.1000133}$
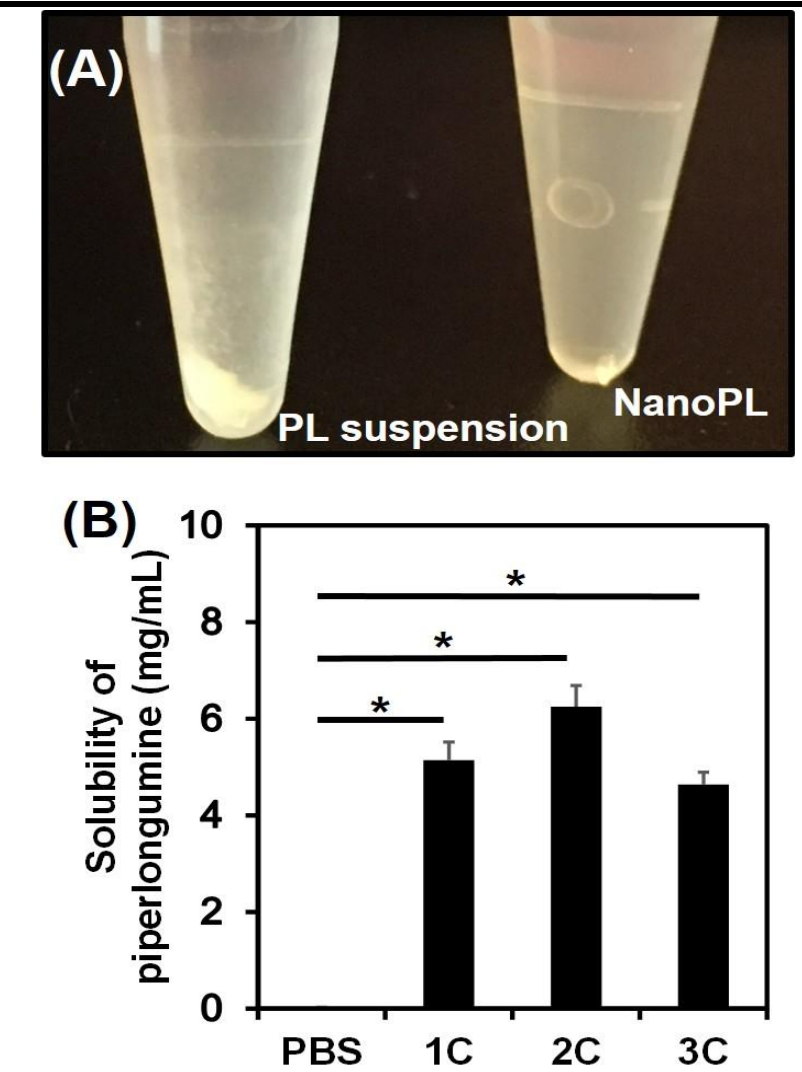

Figure 2: The solubility of PL in PBS ( $\mathrm{pH} 7.4)$ and in liposomal nanoformulations (NanoPLs). (A) PL aggregates can be easily separated from the PL suspension by centrifugation and collected at the bottom of the Eppendorf tube (left). On the contrary, nanoPLs remain a nearly clear colloidal dispersion after centrifugation (right). (B) The solubility of PL in PBS and in NanoPLs determined by HPLC. " $\mathrm{p}<0.05$.

\section{Storage stability of nanoPLs}

The storage stability of NanoPL $1 \mathrm{C}, 2 \mathrm{C}$, and $3 \mathrm{C}$ were evaluated at $4^{\circ} \mathrm{C}$ for 12 weeks. The average particle size and size distribution were measured at 4, 8 and 12 weeks. The EE\% and DL\% were determined after 12 weeks. NanoPL 1C, 2C, and 3C all demonstrated high stability during storage. No significant change in particle size, size distribution, EE\% or DL\% was observed during the test $(\mathrm{p}>0.05$, Figure $3 \mathrm{~A}, 3 \mathrm{~B}$ and Table 2).
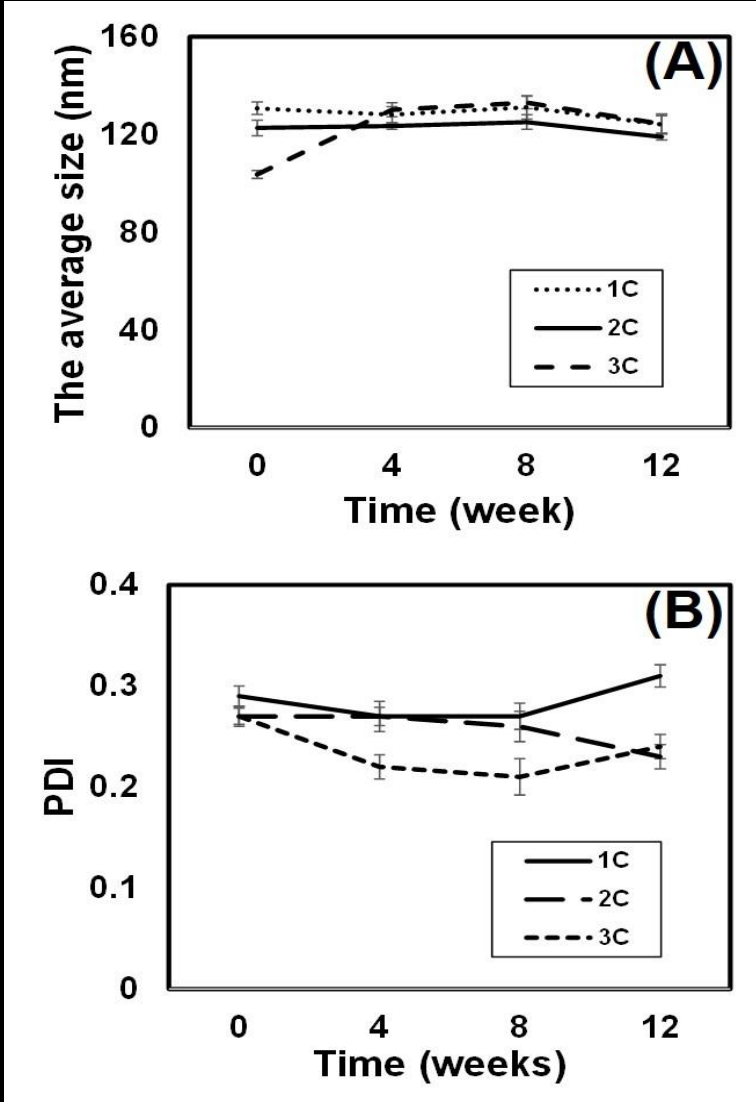

Figure 3: The physical stability of nanoPLs during storage at $4^{\circ} \mathrm{C}$. The particle size and PDI were measured by DLS right after the preparation of nanoPLs and after 12 weeks of storage.

\section{In vitro antitumor activity against human prostate} cancer PC-3 cells

The In vitro antitumor effects of nanoPLs were evaluated using human prostate cancer PC-3 cell line. In order to examine the possible toxicity from the lipid materials, three empty liposomal vehicles containing the same lipid composition as of formulation $1 \mathrm{C}, 2 \mathrm{C}$ and $3 \mathrm{C}$ were prepared. PC-3 cells were exposed to nanoPLs at the final PL concentration of $24 \mu \mathrm{M}$. Control groups were treated by empty liposomal vehicles at the same lipid concentrations. As shown in Figure 4, after 24 hours of treatment, nanoPLs exhibited strong cell killing ability by reducing the cell viability to $53 \%$ or less, whereas empty liposomal vehicles of $1 \mathrm{C}$ and $2 \mathrm{C}$ only slightly affected cells by reducing the cell viability to $88.72 \pm 7.4 \%$ and $87.4 \pm 5.4 \%$. However, after the treatment by empty liposomal vehicles of $3 \mathrm{C}$, the cell viability decreased to $67.54 \pm 1.95 \%$, which indicated that the liposomal vehicle used in NanoPL $3 \mathrm{C}$ indeed was toxic. 
Citation: Alamri A, Huang Y, Zhang H. Liposomal Nanoformulation of Piperlongumine for Improved Aqueous Solubility and Enhanced Anti-tumour Activity In-vitro. Int J Pharm Pharmacol 2018; 2: 133. doi: $\underline{10.31531 / 2581-3080.1000133}$

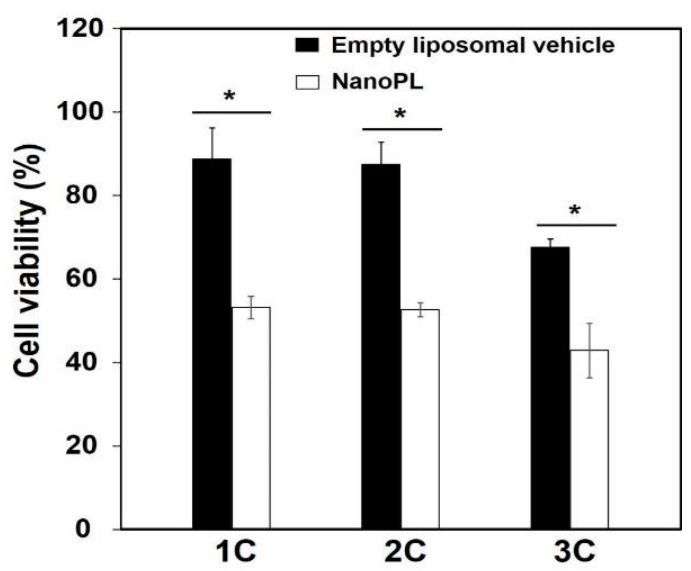

Figure 4: The inhibitory effect of NanoPLs on the growth of PC-3 cells. PC-3 cells were seeded at $1 \times 10^{4}$ cells per well in a 96 well plate 24 hours prior to the treatment. The cells were exposed to free PL and nanoPLs containing $24 \mu \mathrm{M}$ of PL for 24 hours, then the cell viability was determined using a cell proliferation assay kit. ${ }^{*}<0.05$.

After confirming the anti-tumour activity of nanoPLs, we further investigated the dose response. PC-3 cells were treated by free PL and nanoPLs at escalated concentrations from 3 to $30 \mu \mathrm{M}$. We found that the relative percentage of viable cells treated by formulations $1 \mathrm{C}, 2 \mathrm{C}$, and $3 \mathrm{C}$ was significantly lower than that of cells treated by free PL ( $<<0.05$, Figure 5).

At the lower dose of $3 \mu \mathrm{M}$, the viability of the cells treated by free PL was $91.2 \pm 0.93 \%$. At the same dose, the cell viability with liposomal formulations $1 \mathrm{C}, 2 \mathrm{C}$, and $3 \mathrm{C}$ were $93.34 \pm 4.8 \%, 87.98 \pm 6.19 \%$, and $87.76 \pm$ $3.92 \%$, respectively.

The cell viability gradually decreased with the increase of PL concentration. Clear dose-dependent response was observed for both free PL and nanoPLs (Figure 5). After the treatment by $30 \mu \mathrm{M}$ of free PL, the viable cells were $86.93 \pm 3.99 \%$. But the percentage of viable cells was significantly deceased the treatment by nanoPLs at the same dose level of PL. Specifically, the viability were $47.05 \pm 4.83 \%, 41.56 \pm 3.94 \%$, and $28.92 \pm 1.22 \%$, after the treatment by $1 \mathrm{C}, 2 \mathrm{C}$, and $3 \mathrm{C}$ formulations, respectively. To best describe the anti-tumour activity, the IC50 values were calculated for nanoPLs 1C, 2C, 3C and free PL. As shown in Table 3, the IC50 of formulations $1 \mathrm{C}, 2 \mathrm{C}, 3 \mathrm{C}$ were $35.15 \pm 3.27 \mu \mathrm{M}, 29.49$ $\pm 0.03 \mu \mathrm{M}$, and $23.74 \pm 2.05 \mu \mathrm{M}$, respectively, which were significantly lower than that of free PL, which was $45.68 \pm 5.02 \mu \mathrm{M}$.

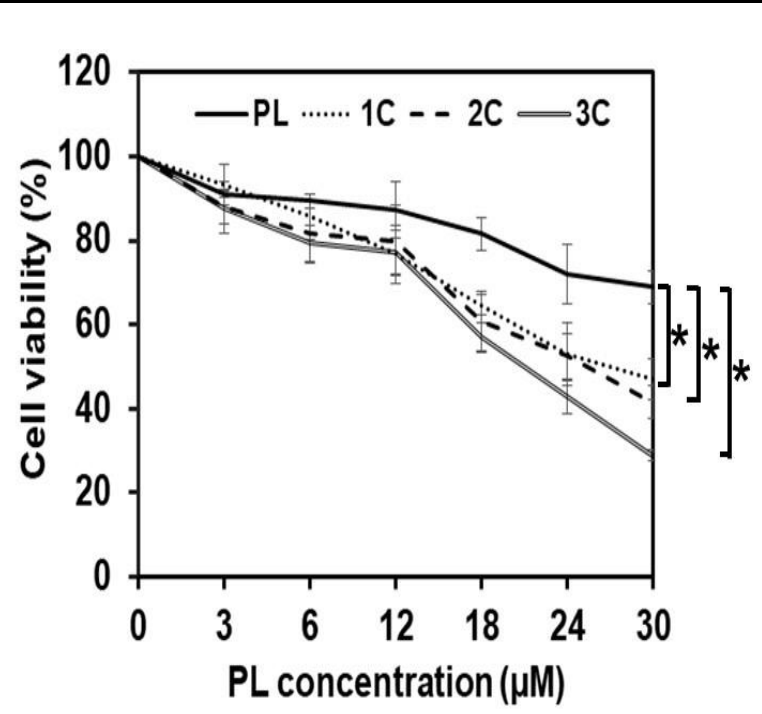

Figure 5: The dose-dependent In vitro anti-tumour activity of NanoPLs. PC-3 cells were seeded at $1 \times 10^{4}$ cells/well in a 96 well plate 24 hours prior to the treatment. The cells were exposed to free PL and nanoPLs at different concentrations $(3,6,12,18,24$ and $30 \mu \mathrm{M}$ ) for 24 hours, then the cell viability was determined using a cell proliferation assay kit. ${ }^{*} \mathrm{p}<0.05$.

Table 3: The IC50 of free PL and NanoPLs to PC-3 cells. [Note: ${ }^{*} \mathrm{p}<0.05$ versus free PL. ${ }^{* *} \mathrm{p}<0.01$ versus free PL. ${ }^{* * *}$ p $<0.005$ versus free PL].

\begin{tabular}{|l|l|}
\hline Sample & IC50 (mM) \\
\hline Free PL & $45.68 \pm 5.02$ \\
\hline $1 C$ & $35.15 \pm 3.27^{*}$ \\
\hline $2 C$ & $29.49 \pm 0.03^{* *}$ \\
\hline $3 C$ & $23.74 \pm 2.05^{* * *}$ \\
\hline
\end{tabular}

\section{Discussion}

With the solubility in PBS ( $\mathrm{pH}=7.4$ ) at only $0.02 \pm 0.01$ $\mathrm{mg} / \mathrm{mL}$ (Figure $2 \mathrm{~B}$ ), PL is a "practically insoluble (PI)" compound based on the solubility classification in the US Pharmacopeia. Although PL has demonstrated several pharmacological activities, especially in cancer treatment, this poor aqueous solubility has largely impeded its clinical investigation and application. Previously reported polymeric nanoparticles and micelles [5,17-19] are valuable tools to improve PL's solubility, but none of the polymer materials have been approved by the US FDA for intravenous administration. The safety and biocompatibility of these 
Citation: Alamri A, Huang Y, Zhang H. Liposomal Nanoformulation of Piperlongumine for Improved Aqueous Solubility and Enhanced Anti-tumour Activity In-vitro. Int J Pharm Pharmacol 2018; 2: 133. doi: $\underline{10.31531 / 2581-3080.1000133}$

polymers in human is still unclear. Liposomal nanoparticles are apparently advantageous over polymeric nanoparticles and polymeric micelles due to the excellent biocompatibility and safety record of lipid materials in human [24]. For example, liposomal formulation Doxil ${ }^{\circledR}$ (liposomal doxorubicin) and Marqibo $^{\circledR}$ (liposomal vincristine) were approved by the US FDA because both products are able to enhance the apparent solubility and therapeutic efficacy of the drug in cancer. Therefore, the primary objective of this study was to develop a liposomal nanoformulation, in which PL is sufficiently soluble and suitable for intravenous administration. We report for the first time that liposomal nanoformulations dramatically improved the aqueous solubility of PL with a very high encapsulation efficiency (Table 2). Specifically, formulation 2C raised the solubility of PL to $6.25 \pm 0.44 \mathrm{mg} / \mathrm{mL}$. Because the volume of general intravenous injection to each mouse is limited to $100 \mu \mathrm{L}$ or less, this markedly elevated PL aqueous solubility makes it very convenient for In vitro anti-tumour experiments using tumour-bearing mouse models, which we have already planned for. It is important to note that even the commercial product Doxil $^{\circledR}$ can only raise the doxorubicin concentration to $2 \mathrm{mg} / \mathrm{mL}$ ) [32] and Marqibo ${ }^{\circledR}$ can only solubilize vincristine to $0.16 \mathrm{mg} / \mathrm{mL}$ [38]. Therefore, the aqueous solubility problem of PL is successfully addressed by using liposomal nanotechnology.

The particle size, size distribution and storage stability are crucial factors for the successful application of liposomal nanoparticles. During the formulation development, the influence of the percentage of DOTAP lipid on the particle size and stability of nanoPLs was evaluated. Our results have shown that higher percentage of DOTAP in the formulation helped reduce the size and enhance the stability of liposome formulations, probably by increasing the fluidity of lipid membrane. This is consistent with the results reported by Campbell and co-workers [39,40]. In addition, DOTAP contributes positive surface charge to nanoPLs, resulting in elevated electro-repulsive tension and reduced the tendency of collision between liposomal nanoparticles. Another key parameter influencing the particle size is the ratio of PL to total lipids. Due to the strong hydrophobicity, PL molecules have to reside in the lipid bilayer of liposomes in order to avoid forming aggregates. When the ratio of PL to total lipids is high, e.g. at $2 / 10$, there are more PL molecules in the lipid bilayer, which increased the rigidity and reduced the curvature of the lipid bilayer. Therefore, the particles prepared at $2 / 10$ ratio are relatively larger than at $1 / 10$ ratio. The excellent stability of nanoPLs should also be attributed, at least in part, to the addition of natural lipid cholesterol [41]. Overall, by rationally controlling the lipid components, particle size and surface charge, nanoPLs (1C, 2C and 3C) developed in the study demonstrated excellent storage stability (Table 2 and Figure 3).

In the last part of this study, the In vitro anti-tumour activity was investigated and the IC50 of free PL and nanoPLs to PC-3 cells were determined. Because nanoparticles greater than $200 \mathrm{~nm}$ in diameter are usually prone to aggregate during storage in buffer, are rapidly cleared in the blood stream, and are less efficient in passive accumulation in solid tumour tissues [42,43], we selected formulations with the average particle size below $200 \mathrm{~nm}$ for cytotoxicity studies. Compare to free PL, nanoPLs exhibited a significantly increased cytotoxicity $(p<0.05)$ (Figures 4 and 5). The strongest antitumor activity was achieved with formulation 3C. The enhanced cytotoxicity can be accounted for the positive surface charge of nanoPLs conferred by DOTAP. Since mammalian cell membrane has a negative surface charge, positively charged liposomes have a high affinity to the cells based on electrostatic interaction $[39,44,45]$.

We investigated whether the enhanced cell killing activity of nanoPLs was caused by the cytotoxicity of liposomal vehicles. Three different empty liposomal formulations were prepared using the same lipid components and composition used in nanoPLs 1C, 2C and $3 \mathrm{C}$, and then PC-3 cells were treated with the empty liposome controls at the same lipid concentration. As shown in Figure 4, empty liposomal vehicles used in formulation $1 \mathrm{C}$ and $2 \mathrm{C}$, which contain relatively lower percentage of DOTAP, showed little toxicity to PC-3 cells.

The results indicated that formulations $1 \mathrm{C}$ and $2 \mathrm{C}$ induced cancer cell death indeed by PL, very likely through enhanced intracellular delivery by cationic liposomes. It should be pointed out that when the DOTAP content was increased to $50 \%$, empty liposomal vehicles used in nanoformulation $3 \mathrm{C}$ did show obvious toxicity to PC-3 cells. We further demonstrated that nanoPLs could elicit a strong antitumour activity in a dose-dependent manner to prostate cancer PC-3 cells (Figure 5). In addition, the IC50 values of nanoPLs were significantly lower than that of free PL (Table 3). Therefore, nanoPLs $1 \mathrm{C}$ and 2C appear to be better choice for In vitro investigation in the future. 
Citation: Alamri A, Huang Y, Zhang H. Liposomal Nanoformulation of Piperlongumine for Improved Aqueous Solubility and Enhanced Anti-tumour Activity In-vitro. Int J Pharm Pharmacol 2018; 2: 133. doi: $\underline{10.31531 / 2581-3080.1000133}$

\section{Conclusion}

In this study, the first liposomal nanoformulation for the natural active compound PL was developed. The aqueous solubility issue of PL was successfully solved using liposomal nanotechnology. NanoPLs demonstrated excellent storage stability and strong antitumour activity In vitro. The results of this study warrant further investigation of nanoPLs in animal models of human prostate cancer as well as other cancer types. NanoPLs provide a "soluble" form of PL in aqueous solution, which can be conveniently prepared by most of the research laboratories and easily used by researchers from a variety of therapeutic areas, such as oncology, cardiology and inflammation, etc. Our results showed that nanoPLs have the potential to dramatically boost the preclinical and clinical investigation of PL.

\section{Conflict of Interest}

The authors declare that there are no conflicts of interest.

\section{Acknowledgements}

This study was financially supported by the Department of Pharmaceutical Sciences and the Faculty Development Committee at MCPHS University. Ali Alamri is thankful to the financial support by the Saudi Arabian Cultural Mission for his study at MCPHS University.

\section{References}

1. Lin Z, Liao Y, Venkatasamy R, et al. Amides from Piper nigrum L. with dissimilar effects on melanocyte proliferation in-vitro. J Pharm Pharmacol 2007; 59: 529-536.

2. Bezerra DP, Pessoa C, de Moraes MO, et al. Overview of the therapeutic potential of piplartine (piperlongumine). Eur J Pharm Sci 2013; 48: 453463.

3. Raj L, Ide T, Gurkar AU, et al. Selective killing of cancer cells by a small molecule targeting the stress response to ROS. Nature 2011; 475: 231-234.

4. Adams DJ, Dai M, Pellegrino G, et al. Synthesis, cellular evaluation, and mechanism of action of piperlongumine analogs. Proc Natl Acad Sci USA 2012; 109: 15115-15120.

5. Liu Y, Chang Y, Yang C, et al. Biodegradable nanoassemblies of piperlongumine display enhanced anti-angiogenesis and anti-tumor activities. Nanoscale 2014; 6: 4325-4337.
6. Wang Y, Wang JW, Xiao X, et al. Piperlongumine induces autophagy by targeting p38 signaling. Cell Death Dis 2013; 4: e824.

7. Bharadwaj U, Eckols TK, Kolosov M, et al. Drugrepositioning screening identified piperlongumine as a direct STAT3 inhibitor with potent activity against breast cancer. Oncogene 2015; 34: 13411353.

8. Aodah A, Pavlik A, Karlage $\mathrm{K}$, et al. Preformulation Studies on Piperlongumine. PLoS One 2016; 11: e0151707.

9. Fofaria NM, Qhattal HS, Liu X, et al. Nanoemulsion formulations for anti-cancer agent piplartine-Characterization, toxicological, pharmacokinetics and efficacy studies. Int J Pharm 2016; 498: 12-22.

10. Wijeyaratne SM, Ubayasiri RA, Weerasinghe C. Fatal pulmonary embolism of polyvinyl alcohol particles following therapeutic embolisation of a peripheral arteriovenous malformation. BMJ Case Rep 2009.

11. Douglas FG, Kafilmout KJ, Patt NL. Foreign particle embolism in drug addicts: Respiratory pathophysiology. Ann Intern Med 1971; 75: 865880.

12. Liebmann J, Cook JA, Mitchell JB. Cremophor EL, solvent for paclitaxel, and toxicity. Lancet 1993; 342: 1428.

13. Gelderblom H, Verweij J, Nooter $K$, et al. Cremophor EL: The drawbacks and advantages of vehicle selection for drug formulation. Eur J Cancer 2001; 37: 1590-1598.

14. Sparreboom A, van Tellingen O, Nooijen WJ, et al. Nonlinear pharmacokinetics of paclitaxel in mice results from the pharmaceutical vehicle Cremophor EL. Cancer Res 1996; 56: 2112-2115.

15. Weiszhar Z, Czucz J, Revesz C, et al. Complement activation by polyethoxylated pharmaceutical surfactants: Cremophor-EL, Tween- 80 and Tween20. Eur J Pharm Sci 2012; 45: 492-498.

16. Szebeni J, Muggia FM, Alving CR. Complement activation by Cremophor EL as a possible contributor to hypersensitivity to paclitaxel: An In vitro study. J Natl Cancer Inst 1998; 90: 300-306.

17. Venkatesan J, Alam MS, Hong EJ, et al. Preparation of piperlongumine-loaded chitosan nanoparticles for safe and efficient cancer therapy. RSC Advances 2016; 6: 79307-79316.

18. Wang J, Yao K, Wang C, et al. Synthesis and drug delivery of novel amphiphilic block copolymers containing hydrophobic dehydroabietic moiety. J Materials Chem B 2013; 1: 2324-2332. 
Citation: Alamri A, Huang Y, Zhang H. Liposomal Nanoformulation of Piperlongumine for Improved Aqueous Solubility and Enhanced Anti-tumour Activity In-vitro. Int J Pharm Pharmacol 2018; 2: 133. doi: 10.31531/2581-3080.1000133

19. Sharkey CC, Li J, Roy S, et al. Two-stage nanoparticle delivery of piperlongumine and tumor necrosis factor-related apoptosis-inducing ligand (TRAIL) anti-cancer therapy. Technol (Singap World Sci) 2016; 4: 60-69.

20. van Hoogevest P, Liu X, Fahr A. Drug delivery strategies for poorly water-soluble drugs: the industrial perspective. Expert Opin Drug Deliv 2011; 8: 1481-500.

21. Fahr A, Liu X. Drug delivery strategies for poorly water-soluble drugs. Expert Opin Drug Deliv 2007; 4: 403-416.

22. Mohammed AR, Weston N, Coombes AG, et al. Liposome formulation of poorly water-soluble drugs: Optimisation of drug loading and ESEM analysis of stability. Int J Pharm 2004; 285: 23-34.

23. Allen TM, Cullis PR. Drug delivery systems: Entering the mainstream. Science 2004; 303: 18181822.

24. Allen TM, Cullis PR. Liposomal drug delivery systems: From concept to clinical applications. Adv Drug Deliv Rev 2013; 65: 36-48.

25. Koudelka S, Turanek J. Liposomal paclitaxel formulations. J Control Release 2012; 163: 322334.

26. Wang-Gillam A, Li CP, Bodoky G, et al. Nanoliposomal irinotecan with fluorouracil and folinic acid in metastatic pancreatic cancer after previous gemcitabine-based therapy (NAPOLI-1): A global, randomised, open-label, phase 3 trial. Lancet 2016; 387: 545-557.

27. Ur Rehman SS, Lim K, Wang-Gillam A. Nanoliposomal irinotecan plus fluorouracil and folinic acid: a new treatment option in metastatic pancreatic cancer. Expert Rev Anticancer Ther 2016; 16: 485-492.

28. Xu Q, Tanaka Y, Czernuszka JT. Encapsulation and release of a hydrophobic drug from hydroxyapatite coated liposomes. Biomaterials 2007; 28: 26872694.

29. Matsumura Y, Maeda H. A new concept for macromolecular therapeutics in cancer chemotherapy: Mechanism of tumoritropic accumulation of proteins and the antitumor agent smancs. Cancer Res 1986; 46: 6387-6392.

30. Maeda H, Wu J, Sawa T, et al. Tumor vascular permeability and the EPR effect in macromolecular therapeutics: A review. J Control Release 2000; 65: 271-284.

31. Clark AJ, Wiley DT, Zuckerman JE, et al. CRLX101 nanoparticles localize in human tumors and not in adjacent, nonneoplastic tissue after intravenous dosing. Proc Natl Acad Sci USA 2016; 113: 3850-3854.

32. Abraham SA, Waterhouse DN, Mayer LD, et al. The liposomal formulation of doxorubicin. Methods Enzymol 2005; 391: 71-97.

33. Harrington KJ, Mohammadtaghi S, Uster PS, et al. Effective targeting of solid tumors in patients with locally advanced cancers by radiolabeled pegylated liposomes. Clin Cancer Res 2001; 7: 243-254.

34. Douer D. Efficacy and safety of vincristine sulfate liposome injection in the treatment of adult acute lymphocytic leukemia. Oncologist 2016; 21: 840847.

35. Zhang H. Thin-film hydration followed by extrusion method for liposome preparation. Methods Mol Biol 2017; 1522: 17-22.

36. Wu S, Sun C, Pei S, et al. Preparative isolation and purification of amides from the fruits of Piper longum L. by upright counter-current chromatography and reversed-phase liquid chromatography. J Chromatogr A 2004; 1040: 193204.

37. Volpe DA, Hamed SS, Zhang LK. Use of different parameters and equations for calculation of $\operatorname{IC}(5)(0)$ values in efflux assays: Potential sources of variability in IC(5)(0) determination. AAPS J 2014; 16: $172-180$.

38. Silverman JA, Deitcher SR. Marqibo(R) (vincristine sulfate liposome injection) improves the pharmacokinetics and pharmacodynamics of vincristine. Cancer Chemother Pharmacol 2013; 71: 555-564.

39. Campbell RB, Balasubramanian SV, Straubinger RM. Phospholipid-cationic lipid interactions: Influences on membrane and vesicle properties. Biochim Biophys Acta 2001; 1512: 27-39.

40. Campbell RB, Balasubramanian SV, Straubinger $\mathrm{RM}$. Influence of cationic lipids on the stability and membrane properties of paclitaxel-containing liposomes. J Pharm Sci 2001; 90: 1091-1105.

41. Kirby C, Clarke J, Gregoriadis G. Effect of the cholesterol content of small unilamellar liposomes on their stability In vitro and In vitro. Biochem J 1980; 186: 591-598.

42. Maruyama K. Intracellular targeting delivery of liposomal drugs to solid tumors based on EPR effects. Adv Drug Deliv Rev 2011; 63: 161-169.

43. Petros RA, DeSimone JM. Strategies in the design of nanoparticles for therapeutic applications. Nat Rev Drug Discov 2010; 9: 615-627.

44. Miller CR, Bondurant B, McLean SD, et al. Liposome-cell interactions In vitro: Effect of liposome surface charge on the binding and 
Citation: Alamri A, Huang Y, Zhang H. Liposomal Nanoformulation of Piperlongumine for Improved Aqueous Solubility and Enhanced Anti-tumour Activity In-vitro. Int J Pharm Pharmacol 2018; 2: 133. doi: $\underline{10.31531 / 2581-3080.1000133}$

endocytosis of conventional and sterically stabilized liposomes. Biochemistry 1998; 37: 12875-12883.
45. Campbell RB, Ying B, Kuesters GM, et al. Fighting cancer: From the bench to bedside using second generation cationic liposomal therapeutics. J Pharm Sci 2009; 98: 411-29.

This manuscript was peer-reviewed

Mode of Review: Single-blinded

Academic Editor: Dr. Syed Sarim Imam

Copyright: (C2018 Alamri, et al. This article is distributed under the terms of the Creative Commons Attribution 4.0 International License (http://creativecommons.org/licenses/by/4.0/), which permits unrestricted use, distribution, and reproduction in any medium, provided you give appropriate credit to the original author(s) and the source, provide a link to the Creative Commons license, and indicate if changes were made. 